\title{
HEARING THE HEAVENS
}

The cosmos is thought to be awash with gravitational waves to which humanity is, as yet, deaf. Trudy E. Bell reports on LISA, an experiment on an unprecedented scale designed to put that right. 
tion magine a new constellation - a narrow triangle about as deep as the scoop of the Big Dipper. But this constellation, unlike the familiar natural ones, moves through the sky, always appearing in the evening sky after sunset. The new constellation slowly rotates, each component circling around the centre once every year. And as it does so, it also expands and contracts.

Unaided earthly eyes will never actually see the Laser Interferometer Space Antenna (LISA), as this artificial constellation is to be named. Its three component spacecraft will be too small, and the light with which they shine will be invisible infrared. Unseen as it may be, though, it will still be humanity's largest ever creation -5 million kilometres on a side. And the aspirations it embodies are similarly grandiose. In 2018, or thereabouts, it will try to detect extraordinary cosmic events, such as the births of galaxies and the deaths of black holes, in a fundamentally new way.

LISA is perhaps the most ambitious space mission envisaged for the coming decades. A recent assessment of astrophysics research proposed for NASA's Beyond Einstein programme by America's National Research Council (NRC) ${ }^{1}$ gave the "extraordinarily original and technically bold" project its highest scientific ranking. Under joint development by NASA and the European Space Agency (ESA), LISA's goal is to detect gravitational waves - fluctuations in the fabric of space and time - by measuring the relative motions of three spacecraft with great precision. Although that assessment carried out by the NRC saw LISA as the "least scientifically risky" of the proposals for future flagship missions, there is no getting away from the fact that no one has ever tried anything remotely similar before.

LISA is designed to measure the gravitational waves predicted by Einstein's general theory of relativity. Relativity defines gravity in terms of the geometry of space and time; gravitational waves are transient fluctuations that stretch and compress that geometry. Although they have never yet been observed directly, indirect evidence for them was enough to earn a Nobel Prize. Over three decades Russell Hulse and Joseph Taylor, now of Princeton University, New Jersey, observed a system in which two neutron stars whirl round each other once every 8 hours. Over the years, their spinning speeded up - evidence that the system was losing energy. The rate at which energy was lost matched exactly the power with which relativity predicted that the pair of stars would be emitting gravitational waves.

\section{Geodesics and gigantism}

Even when gravitational waves are generated by massive objects and ungodly accelerations, such as those involved in that neutron-star doublet, detecting them requires almost unthinkable attention to detail. In relativity, the shape of spacetime is defined as the path taken by a body falling free under no influence but that of gravity - a 'geodesic'. Gravitational waves affect these geodesics, and can thus be detected; but only through careful comparisons. "You cannot demonstrate that one particle is freely falling and following a geodesic," explains Stefano Vitale, a physics professor at the University of Trento in Italy. "You must have two particles, and compare the shapes of their paths by exchanging a ray of light between them." The small amplitude of gravitational waves means that detecting them by comparing geodesics in this way requires immense precision. The long wavelengths of the gravitational waves that most interest astrophysicists means that the geodesics being compared must be separated from each other by astronomical distances.

Using freely falling objects to spot gravitational waves in this way, as LISA is intended to do, is a three-step process. First you set up a situation in which masses can fall freely along their geodesics without being disturbed by magnetic fields or other spurious forces. Then you must measure with extraordinary precision how the distance between their geodesics changes when passing gravitational waves distort the local curvature of space. The last step is analysing these changes to determine the exact shape, frequency and intensity of the distortions to the curvature of space, so as to learn about the nature of distant events producing them.

To provide Vitale's geodesic-joining rays of light, LISA uses neodymium-YAG lasers, which shine at a wavelength of a little more than a micrometre, a wavelength they stick to with extreme precision. These will illuminate cubes four kilograms in mass but just four centimetres on a side - polished 'test masses' of gold-coated gold-platinum alloy as beautiful as fine jewellery and much more costly. The test masses respond to gravity and not much else. "The goldplatinum alloy has a magnetic susceptibility almost as low as glass," explains Paul McNamara, an ESA project scien-
"We hope to use gravitational waves to peer through this dark fog to listen to the birth of the first galaxies." - Scott Hughes tist in Noordwijk, the Netherlands. Once in space, the test masses' sole purpose is to follow their own paths, each falling freely along its geodesic within one of the LISA spacecraft while reflecting the laser light with which the other spacecraft illuminate it.

LISA will be arranged so that these geodesics are 5 million kilometres apart, with the spacecraft falling around the Sun $20^{\circ}$ behind the Earth in the same orbit. That will put them about 50 million kilometres from their planet of origin. Once during every annual orbit the triangle will 'breathe', its sides taking turns to grow and shrink by about 50,000 kilometres.

\section{Hearing double}

LISA's spacecraft do not need to measure the exact distance between the test masses each contains any more than a police officer with a radar gun needs to know the exact distance to a car before he can tell whether it is speeding. The system's 'breathing' causes a predictable Doppler shift - similar to the lowered tone of a receding train's whistle, or the redshift of a distant galaxy's light - in the light reflected from the test mass in one spacecraft to the instruments in another when the distance between them grows, and an opposite shift on contraction.

A passing gravitational wave will impose a variation on this predictable Doppler signal, because it will curve space between spacecraft and lengthen the route that the light connecting them has to take. Compared with the changes in the Doppler shift caused by the breathing, however, frequency changes due to gravitational waves happen much faster - over periods of between 10 seconds and 3 hours. It is here that the unprecedented precision of LISA's metrology will come into play. LISA's detectors will treat the infrared laser beam in the same way that an FM radio in a car treats a radio signal. An FM radio station transmits a steady highfrequency signal (typically around 100 megahertz, or $10^{8}$ cycles per second) modulated by lower-frequency audio programming (in the kilohertz range, or about $10^{3}$ cycles per second). A home or car radio receiver contains a local oscillator that generates a signal at roughly the same higher frequency as the transmitter. Subtracting the signal received over the air from the local oscillator signal, a process called heterodyning, unmasks the much lower-frequency signal of the audio programming. That audio-frequency signal then drives the radio's speakers.

Optical heterodyning within LISA, using the milliontimes higher frequencies of infrared laser light (about 

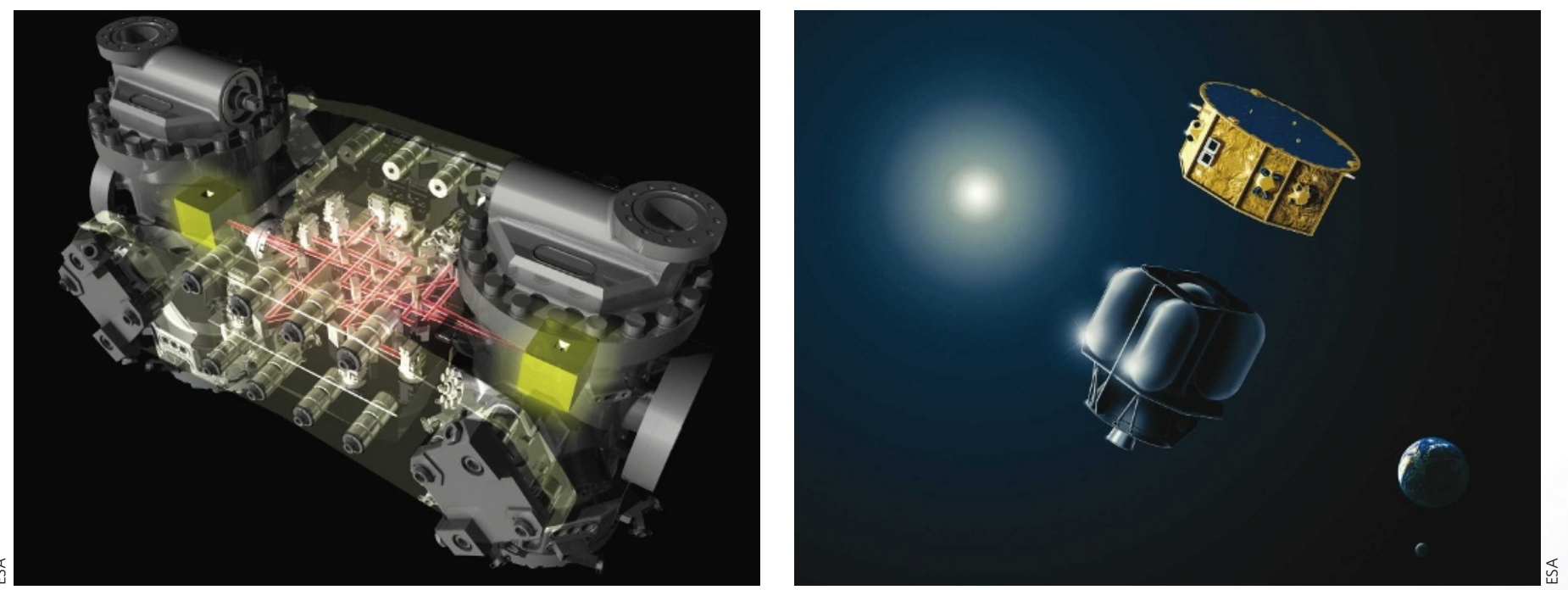

300 terahertz $-3 \times 10^{14}$ cycles per second), will do much the same but with unprecedented precision. The incoming laser beam (as reflected off a distant test mass) will be combined with some light from the outgoing laser beam (treated as the reference signal) on a photodetector. The comparison will reveal the expected lower-frequency (1 megahertz) 'hum' of LISA's regular breathing. Gravitational waves would show up as a modulation of that hum at really low frequencies (a millihertz, or 0.001 cycles per second). Using signal processing akin to that in satellite-navigation receivers, LISA will measure that low-frequency signal to a precision of a few millionths of a cycle, corresponding to a few picometres in distance.

\section{Finding the path}

Unsurprisingly, some wonder whether this wizardry can be pulled off in practice. Specifically, can external disturbances - for example, the solar wind and the radiation pressure of the Sun's light - be understood and avoided well enough for LISA to achieve the sensitivity it needs?

"The devil is truly in the details," cautions Robin Stebbins, LISA project scientist at NASA's Goddard Space Flight
LISA Pathfinder in artists' impressions with test masses and laser light revealed in cutaway (left) and reaching its Lissajous orbit (right).

\section{Meanwhile, back on Earth ...}

LISA is not the only gravitational wave detector: there are five laser interferometers at various stages of development and operation on Earth's surface, although they have yet to detect anything - and may never do so. The Laser Interferometer GravitationalWave Observatory (LIGO) consists of a pair of detectors in Hanford, Washington and a third in Livingston, Louisiana, in the United States. Geo the name is simply a reminder that the detector is located on Earth - sits near Hannover, Germany. Virgo - not an acronym but the name of a constellation that contains a cluster of active galaxies predicted to be a major source of gravitational waves - is near Pisa, Italy. Tama is named after a western suburb of Tokyo, Japan, where the detector is located. AIGO, the Australian International Gravitational Observatory, is in Gingin near Perth, Western Australia. Because they are on Earth, these detectors are much smaller than LISA, with arm lengths that range from 300 metres to 4 kilometres, and thus are sensitive only to short wavelength (high-frequency) signals. Their sensitivity is impaired by seismic noise issues that LISA will avoid, although LISA has its own noise challenges. Ground-based detectors at current levels of sensitivity are not expected to detect gravitational waves because of the rarity and weakness of the stellarmass gravitational-wave sources found at high frequencies; however, planned improvements (specifically Advanced LIGO, to be finished in 2013) will increase sensitivity by more than a factor of 10. At that point some high-frequency detections are expected - if the cosmos plays ball.

T.E. B.
Center in Greenbelt, Maryland. "When doing precision measurements, an instrumentalist must estimate all possible errors, verify in the lab that those estimates are correct, and constantly search for errors that may have been overlooked. We have a list of about 50 physical phenomena that could disturb LISA's test masses, of which perhaps 35 are significant."

There is, however, nothing as convincing as actually trying the technology out. That's where LISA Pathfinder comes in. LISA Pathfinder, currently being assembled by contractor EADS Astrium in Stevenage, UK, is a spacecraft designed to demonstrate LISA's underlying technologies: reflecting infrared laser beams off freely falling masses following their own geodesics, picometre metrology, and reducing unwanted disturbances to the test masses. "Flight hardware is being delivered almost every day for launch in 2010," says McNamara, who is LISA Pathfinder's project scientist.

\section{Lissajous orbit}

LISA Pathfinder will be launched into an elaborate Lissajous (pronounced, by coincidence, "Lisa-ju") orbit around the ' $\mathrm{L}-1$ ' point 1.5 million kilometres sunwards of Earth, where the pull of the Earth and Sun mostly cancel each other out. "We want to get out of the gravitational pull of the Moon or away from the temperature drop of being eclipsed by Earth's shadow," McNamara says. "L-1 has constant solar power and constant temperature, and is relatively cheap to get to."

Once the spacecraft is in this orbit, two test masses just like those to be used in LISA will be gently released from the cages that have held them in place. This is no easy task: "A caging mechanism that would release the cubes with zero velocity was one of the toughest engineering problems - we've been working on it since '94," says McNamara. Once released the test masses, just like the ones in LISA, will fall freely along their own geodesics; only they'll do so separated by a few tens of centimetres, rather than a few million kilometres.

The rest of the LISA Pathfinder spacecraft will serve to shield the masses within from several dozen forces that would otherwise disturb any measurements. Like a parent holding an umbrella over a child in a rainstorm, the spacecraft will follow the test masses without touching them, continuously correcting its position by firing micronewton thrusters so gentle that their force is "the same as your breath from 200 metres away", McNamara says. If Pathfinder can protect its test masses, leaving them to fall 
as freely as if they were the only things in the Universe, then LISA's three spacecraft should be able to do the same.

LISA Pathfinder - a single spacecraft with a 90-day mission quite close to Earth - has a budget of $€ 300$ million (\$440 million). LISA itself - three spacecraft with a 5- to 10-year lifetime, optical heterodyning equipment, deepspace communications and additional intricacies - is put at around US $\$ 2$ billion. The cost is one reason why LISA is being pursued jointly by NASA and ESA, and why it has taken so long to get into space. "To get any mission into space, one must first convince the space community of its scientific value, because space is a zero-sum game," explains Karsten Danzmann, director of the Albert Einstein Institute in Hannover, Germany, and co-chair of the LISA science team. "In the early 1980s [when ideas for a mission such as LISA were first mooted] the astronomers and planetary scientists had neatly divided the cake. No one wanted to share the cake with the gravitational-wave physicists because it would mean getting a narrower slice. Also, no one at the time could see how to measure such small changes over such vast distances."

LISA made its first formal appearance in 1992, when ESA issued a request for proposals for medium-sized scientific missions and Danzmann and his colleagues - about half of them in the United States - proposed a version of the idea with six small spacecraft. The European agency had taken the lead because NASA was more focused on repercussions from the space shuttle Challenger accident and the faulty mirror aboard the Hubble Space Telescope. But by 1997, NASA and ESA were anticipating a joint mission through coordinated studies of the concept that are still under development today.

\section{Screaming stars}

So if LISA finally starts to resonate with the Universe's gravitational radiation at the end of the next decade, as is currently planned, what new science will be revealed? Making a presentation to the NRC panel last year, Scott Hughes, a physicist at the Massachusetts Institute of Technology in Cambridge, provided a striking answer. Without warning, he played an audio recording of a tiger attacking and devouring a live monkey. As the monkey's screams echoed throughout the auditorium, "every primate in the room came to attention", recalls Stebbins.

Hughes was demonstrating just how much one can learn from sound as a way of emphasizing the extent to which LISA's unique output can be thought of in terms of noise, rather than vision. The analogy between LISA and human hearing "works surprisingly well”, he says. A pair of star-sized black holes spiralling into each other would be expected to radiate gravitational waves of frequencies in human audio range. If human ears were sensitive to gravitational waves, the final merging of the black holes would sound like a rising warbling that ends in a mouse squeak, if not quite a monkey scream. That final squeak is what gravitational-wave detectors on Earth are trying to hear (see 'Meanwhile back on Earth').

At the much longer wavelengths to which LISA is sensitive, merging supermassive black holes within star clusters would emit a rising roar "more energetic than all the stars in all the galaxies", Stebbins adds. And the source of the waves should be apparent through LISA rather as the direction of a tiger's kill is obvious to a listener in the jungle. Just as with two human ears, "you can tell roughly where a sound originates, because the sound wave hits one ear before the other", says Hughes, so the distance between the LISA spacecraft allows any waves that it detects to be traced back towards

their source. The three LISA spacecraft, 13 times as far from each other as the Moon is from Earth, will be 17 seconds from each other at the speed of light - which is also the speed of gravitational waves. Time lags in the signals measured across the different edges of the triangle should allow LISA to discern the approximate direction of a source of gravitational waves, in part so radio and optical telescopes could take a look. It is a triangle that triangulates.

\section{The infancy of the Universe}

LISA is expected to hear back to when the Universe was younger than a billion years old. "Half a billion years after the Big Bang was the cosmic dark ages, when the Universe was filled with neutral hydrogen gas that absorbed photons," Hughes explains. "We hope to use gravitational waves to peer through this dark fog to listen to the birth of the first galaxies." (In speech, LISA scientists go back and forth between auditory and visual metaphor.)

In addition, there will be a constant background "hissing and crackling" of higher-frequency gravitational

"Gravitational waves will throw open doors to an entirely new and vast vista of the Universe." - Peter Bender waves from " 20 million more ordinary white-dwarf star binaries that fill our galaxy," Danzmann says. But there will be deeper tones, too - notably, the booming collisions of entire galaxies. LISA's sensitivity to such events is one reason for being sure there is something for LISA to hear. "There are supermassive black holes of a million or 10 million solar masses in the centre of virtually every galaxy. And we see from photographs that galaxies are colliding everywhere," says Rainer Weiss, an emeritus physics professor at the Massachusetts Institute of Technology. "So at the wavelength range of LISA, we know there are sources."

The project's promise goes beyond astronomy into fundamental physics. "Since the beginning of the Copernican revolution, astronomy has posed challenges to physics. And we've used physics to understand astronomical objects," said Charles Kennel, a former NASA associate administrator, now director of the Scripps Institution of Oceanography in San Diego, California, who served as co-chair of the NRC report. "LISA will be able to find merging black holes and test general relativity in the strong field limit." That's a relativist's term for situations where gravity is so powerful - that is, the geometry of space is so sharply curved - that phenomena that cannot be observed in any laboratory, or by any observations of nearby astrophysical objects, come into play.

Relativity's predictions about this limit could be tested, though, by observing how matter and the fabric of space and time are wrenched apart near supermassive black holes in the centres of galaxies. General relativity predicts that when smaller black holes, with masses of ten times the Sun's, fall into these much vaster monsters, the geometry of space and time is also being wrenched and kinked in ways never seen anywhere more mundane - and the specifics of the gravitational waves given off should reveal the theoretical limits of general relativity. Only at the edges of masses capable of such acts of cosmic cannibalism - second in violence only to the Big Bang itself - are gravitational waves anticipated to reveal the theoretical limits of general relativity.

"People often say that gravitational waves will open a new window for astronomy," says Peter Bender, a physicist at the University of Colorado's institute JILA in Boulder. "More accurately, gravitational waves will throw open whole doors onto an entirely new and vast vista of the Universe."

Trudy E. Bell is the author of a dozen books on astronomy, space science, history and cycling.

1. Committee on NASA's Beyond Einstein Program, NASA's Beyond Einstein Program: An Architecture for Implementation (Natl Acad. Press, Washington DC, 2007). 